\title{
Comparative Analysis of the Capacity of Tombusvirus P22 and P19 Proteins to Function as Avirulence Determinants in Nicotiana species
}

\author{
Carlos A. Angel, ${ }^{1}$ Yi-Cheng Hsieh, ${ }^{2}$ and James E. Schoelz ${ }^{1}$ \\ ${ }^{1}$ Division of Plant Sciences, University of Missouri, Columbia, MO 65211, U.S.A.; ${ }^{2}$ Department of Plant Pathology and \\ Microbiology, Texas A\&M University, College Station, TX 77843, U.S.A.
}

Submitted 20 April 2010. Accepted 25 September 2010.

\begin{abstract}
We have used an agroinfiltration assay for a comparative study of the roles of tombusvirus P22 and P19 proteins in elicitation of hypersensitive response (HR)-like necrosis and the role of P19 in silencing suppression in Nicotiana species. The advantage of agroinfiltration rather than expression in plant virus vectors is that putative viral avirulence proteins can be evaluated in isolation, eliminating the possibility of synergistic effects with other viral proteins. We found that tombusvirus P22 and P19 proteins elicited HR-like necrosis in certain Nicotiana species but, also, that Nicotiana species could recognize subtle differences in sequence between these proteins. Furthermore, Nicotiana species that responded with systemic necrosis to virion inoculations responded to agroinfiltration of tombusvirus P19 with a very weak and delayed necrosis, indicating that the rapid HR-like necrosis was associated with putative resistance genes and a plant defense response that limited the spread of the virus. Tombusvirus P19 proteins also appeared to differ in their effectiveness as silencing suppressors; in our assay, the P19 proteins of Cymbidium ringspot virus and Tomato bushy stunt virus were stronger silencing suppressors than Cucumber necrosis virus P20. Finally, we show that agroinfiltration can be used to track the presence of putative plant resistance genes in Nicotiana species that target either tombusvirus P19 or P22.
\end{abstract}

Plant virologists have always considered the genus Nicotiana valuable for characterization of viruses. Nicotiana species such as $N$. benthamiana or $N$. clevelandii have been used for propagation of viruses because they are susceptible to a large variety of them. Other Nicotiana species have been used for categorization of viruses through the local lesion response, i.e., the formation of necrotic or chlorotic local lesions in inoculated leaves. With the advent of the gene-for-gene theory (Flor 1971), it became apparent that local lesions might be valuable for understanding plant defenses. According to the gene-forgene theory, a necrotic or chlorotic lesion might form because the product of a plant resistance $(R)$ gene recognizes a viral

Current address for Y. Hsieh: Center for Biosystems Research, University of Maryland, 5115 Plant Sciences Building, College Park, MD 207424450, U.S.A.

Corresponding author: J. E. Schoelz; Telephone: +1.573.882.1185; Fax: +1.573.882.0588; E-mail: schoelzj@missouri.edu

* The $e$-Xtra logo stands for "electronic extra" and indicates that four supplementary figures are published online. avirulence $(A v r)$ gene product; this theory was proven for viruses when the first viral $A v r$ genes were identified (Daubert et al. 1984). A decade later, the first virus $R$ gene was cloned, and it subsequently became possible to identify $A v r-R$ gene combinations (Whitham et al. 1994).

All categories of virus proteins (e.g., replicase, coat protein, movement protein) have been shown to have the capacity to act as Avr determinants (Kang et al. 2005; Schoelz 2006), and a variety of techniques have been used to identify viral Avr determinants, including gene swaps between virulent and avirulent isolates (Schoelz et al. 1986), expression of viral Avr genes in virus vectors (Scholthof et al. 1995a), stable transformation of plants with viral Avr determinants (Culver and Dawson 1991), and Agrobacterium infiltration (agroinfiltration) of viral Avr determinants in resistant hosts (Erickson et al. 1999). With each of these techniques, the assumption is that a putative viral Avr protein will trigger an HR-like necrosis in a cell that carries the cognate $\mathrm{R}$ product, regardless of how the viral $A v r$ genes are expressed. Of all of the techniques, agroinfiltration offers several unique advantages. First, putative viral Avr proteins can be studied in isolation, eliminating the possibility of synergistic effects with other viral proteins derived from either the native virus or a viral vector. Second, agroinfiltration facilitates comparisons of variants of the same viral Avr gene, derived from either different viruses within a family or through mutation of the coding sequence. Third, the agroinfiltration technique could be an ideal method for screening for the presence of the matching $R$ gene in a diverse array of species.

In this paper, we have utilized agroinfiltration to investigate the properties of two putative Avr proteins of Tomato bushy stunt virus (TBSV), the type member of the Tombusviridae. Tombusviruses are associated with some economically important crop diseases, specifically affecting tomato, eggplant, pepper, carnation, and lettuce in the greenhouse and field (Gerik et al. 1990; Obermeier et al. 2001; Yamamura and Scholthof 2005). Their genome is composed of single-stranded positive sense RNA approximately 4,700 to 4,800 nucleotides in length that is encapsidated into isometric virions 30 to $35 \mathrm{~nm}$ in diameter. The TBSV genome is organized into six open reading frames (ORF) (Hearne et al. 1990; Yamamura and Scholthof 2005). The first ORF is translated directly from the genomic RNA to produce a $33-\mathrm{kDa}$ protein $(\mathrm{P} 33)$ and a read-through mechanism leads to second protein of a $92 \mathrm{kDa}$ (P92), which is a viral RNA-dependent RNA polymerase (Nagy and Pogany 2008; White and Nagy 2004). Both P33 and P92 are required for replication and in a ratio of approximately 20 to 1 (Scholthof et al. 1995b). The third ORF is translated from a subgenomic RNA and encodes the 41-kDa capsid or coat protein (P41), 
which is also involved in movement (Desvoyes and Scholthof 2002; Qu et al. 2002). The fourth and fifth ORF are nested, translated from a second single subgenomic RNA and encode two proteins, P22 and P19, respectively (Hearne et al. 1990; Scholthof et al. 1995c). The P19 and P22 proteins have roles in cell-to-cell and long-distance movement, host determination, and symptom development (Scholthof et al. 1995a and c). The P19 protein is also known by its strong suppressor activity of post-trancriptional gene silencing (Scholthof 2006; Voinnet et al. 1999). A small sixth ORF at the $3^{\prime}$ distal end of the TBSV genomic RNA encodes for a small protein designated $\mathrm{pX}$, which is not essential for replication but is required for virus accumulation (Scholthof and Jackson 1997).

The TBSV P22 and P19 proteins have been shown to function as Avr determinants when expressed from a Potato virus $X$ (PVX) vector. A PVX-p22 construct elicited necrotic lesions in $N$. glutinosa and its derived amphidiploid species $N$. edwardsonii, whereas a PVX-p19 construct induced necrotic local lesions in N. tabacum (Scholthof et al. 1995a). The presence of $p 19$ or $p 22$ delayed the movement of the PVX vector out of the inoculated leaf. However, over time the p19 and p22 coding sequences were deleted from the PVX vector, such that the deletion mutant was able to escape the local lesions on the inoculated leaf to infect the rest of the plant. By contrast, the PVX vector with no TBSV insert was able to spread systemically in these three Nicotiana species, inducing a mild mosaic symptom. The implication of these studies is that these TBSV proteins interact with $R$ gene proteins, resulting in the activation of plant defense responses that limit the spread of the virus. In addition, the TBSV P19 protein played a role on development of a severe systemic necrosis symptom in $N$. clevelandii and $N$. benthamiana when expressed from the PVX vector, a symptom that was similar to the wild-type TBSV (Scholthof et al. 1995a). A subsequent paper indicated that the systemic necrosis symptom in $N$. benthamiana was dependent on both the P33 and P19 proteins (Burgyán et al. 2000).

In our paper, we have examined two questions. First, do variations in sequence affect the functions of putative tombusvirus Avr proteins? Second, can agroinfiltration of viral Avr genes be used to track the inheritance or evolutionary history of tombusvirus $R$ genes? Although the answer to this second question may seem, at first glance, to be straightforward, anomalies have been observed in comparing agroinfiltration results to inoculations of viruses. For example, the Tobacco mosaic virus (TMV) replicase is responsible for triggering an HR upon agroinfiltration into $N$. tabacum carrying the $N$ gene but does not trigger an HR upon coagroinfiltration with the $N$ gene into $N$. benthamiana, even though the transgenic $N$. benthamiana respond to the virus with HR (Bhattacharjee et al. 2009). Consequently, it is important to investigate whether putative $R$ gene products would respond with HR to their cognate tombusvirus Avr proteins in a variety of genetic backgrounds. The knowledge gained from this system can provide the groundwork for a survey of Nicotiana species for the presence of $R$ genes effective against tombusvirus $p 19$ and $p 22$ genes.

\section{RESULTS}

\section{Development of HR in Nicotiana species \\ after agroinfiltration of TBSV $p 22$ and $p 19$ genes.}

To determine if agroinfiltration of TBSV $p 22$ and $p 19$ genes would trigger HR in selected Nicotiana species, we used polymerase chain reaction (PCR) to amplify the DNA segment that encodes the $p 22$ and $p 19$ genes, cloned the DNA segment into the Agrobacterium binary vector pKYLX7 (Schardl et al. 1987), and subsequently, mobilized the clone into Agrobacte- rium tumefaciens AGL1. Since the $p 19$ gene is entirely nested in the p22 gene (Hearne et al. 1990), agroinfiltration of a single construct would theoretically express both proteins (Fig. 1). In fact, agroinfiltration of the TBSV nested $p 19$ and $p 22$ gene construct yielded an HR in N. glutinosa, N. edwardsonii, and $N$. tabacum within 3 to 4 days postinfiltration (dpi), with infiltrated tissue becoming completely desiccated by 7 dpi (Fig. 2; Table 1).

To investigate which viral protein triggered HR in which Nicotiana species, we modified the start codons of the $p 19$ and p22 genes to create constructs that would express only the P22 protein, only the P19 protein, or neither protein (Fig. 1). The construct that expressed only $p 22$ triggered HR in N. edwardsonii and $N$. glutinosa but not in N. tabacum (Fig. 2; Table 1). Since $N$. edwardsonii is an amphidiploid derived from N. glutinosa and $N$. clevelandii (Christie et al. 1969), it is likely that a single $R$ gene product, derived from $N$. glutinosa, is responsible for triggering HR in response to TBSV P22. By contrast, the construct that only expressed P19 triggered HR in $N$. tabacum but not in N. edwardsonii and N. glutinosa (Fig. 2; Table $1)$. The construct that contained mutations in the start codons of both p19 and p22 failed to elicit HR in any of the three Nicotiana species. These agroinfiltration results are in agreement with the expression of P19 and P22 proteins through a PVX vector (Scholthof et al. 1995a), in which it was originally shown that the P22 protein triggers HR in $N$. glutinosa and the P19 protein triggers HR in N. tabacum.

In a previous study, TBSV P19 protein expressed from a PVX vector had been shown to elicit a systemic necrosis symptom in $N$. benthamiana and $N$. clevelandii, two Nicotiana species that are also highly susceptible to the TBSV virus (Scholthof et al. 1995a). It was also shown that inoculation of TBSV virions onto either of these plants induced an apical wilt, followed by widespread necrosis and death of the plant in as little as 15 to $20 \mathrm{dpi}$, depending on environmental conditions. Agroinfiltration of TBSV p22 did not elicit any response in either $N$. benthamiana or N. clevelandii (Fig. 2; Table 1), whereas agroinfiltration of TBSV $p 19$ resulted in the development of a weak necrosis symptom in both hosts, developing in some infiltrated panels at approximately $10 \mathrm{dpi}$ (data not shown). The development of this necrosis symptom was enhanced by environmental conditions of low light, but even then, not every infiltrated leaf panel developed necrosis. Given that agroinfiltration of just the pKYLX7 vector under low-light conditions sometimes resulted in necrosis, we considered the necrosis associated with P19 in $N$. clevelandii and $N$. benthamiana a nonspecific reaction to the A. tumefaciens. By contrast, p19 infiltrated into N. tabacum leaf panels, included in the same test, consistently triggered HR necrosis at 2 to 3 dpi (Fig. 2).

To investigate whether a tombusvirus $R$ gene introgressed into $N$. clevelandii had the capacity to respond to agroinfiltration of TBSV proteins with HR, we agroinfiltrated the TBSV p22 and p19 genes into $N$. clevelandii line 36, an $N$. clevelandii addition line in which the resistance to tombusviruses present in $N$. glutinosa was introgressed into $N$. clevelandii. Line 36 contains the full genome of $N$. clevelandii as well as a chromosome pair from $N$. glutinosa and responds with HR to inoculation of the tombusviruses TBSV, Cucumber necrosis virus (CNV), Cymbidium ringspot virus (CymRSV), Lettuce necrotic stunt virus, and Carnation Italian ringspot virus (Schoelz et al. 2006). Line 36 was agroinfiltrated with the constructs expressing P22 or P19 individually as well as the empty vector pKYLX7, and only leaf panels agroinfiltrated with the p22 construct developed HR, usually within 2 to 3 dpi (Fig. 2). No other symptom was observed in leaf panels agroinfiltrated with $p 19$ or pKYLX7 up to $10 \mathrm{dpi}$. This experiment showed that $N$. clevelandii containing an introgressed resistance gene 
from $N$. glutinosa would respond to agroinfiltration of $p 22$ in exactly the same manner as in $N$. glutinosa or $N$. edwardsonii.

\section{The CNV P21 and CymRSV P22 proteins also elicit HR} in $N$. glutinosa and $N$. edwardsonii.

Two lines of evidence suggested that the $R$ gene product that recognized the TBSV P22 protein also recognized the P22 proteins of other tombusviruses. First, $N$. clevelandii line 36 responded to CNV and CymRSV with HR (Schoelz et al. 2006), indicating that, at a minimum, the $R$ gene that recognized these viruses was on the same chromosome as the $R$ gene that recognized TBSV. Second, silencing of the $N$ family of resistance genes compromised the resistance response of $N$. edwardsonii to TBSV, CNV, and CymRSV (Balaji et al. 2007), indicating that the $R$ genes effective against these three tombusviruses must share some degree of sequence homology. Taken together, these studies could not prove that a single $R$ gene product recognized the $\mathrm{P} 22$ protein of all three tombusviruses, but the $R$ gene or genes must be located on the same chromosome and must be related at the nucleotide sequence level.

To further investigate the HR directed against tombusviruses, we examined whether the P22 proteins of CNV and CymRSV would trigger an HR in N. glutinosa and N. edwardsonii in an agroinfiltration assay. For each virus, four types of constructs were developed for agroinfiltration, according to the design in Figure 1. One construct contained the nested sequences of the CymRSV $p 22$ and $p 19$ genes or, for CNV, the analogous $p 21$ and $p 20$ genes. A pair of constructs was also created in which the start codons were mutated such that only one or the other of the proteins would be expressed. In the fourth construct, we mutated both start codons such that neither protein would be expressed.

Agroinfiltration of the CNV p21/p20 nested genes yielded an HR in $N$. edwardsonii and $N$. glutinosa within 3 to $4 \mathrm{dpi}$, a response that was comparable to that elicited by the TBSV nested construct (Table 1). Agroinfiltration of the construct that expressed only the CNV p21 gene yielded the same responses as the CNV nested construct, whereas the construct that expressed the CNV p20 gene and the double mutant did not induce any response in $N$. edwardsonii or $N$. glutinosa. Furthermore, agroinfiltration of CNV p21 did not yield an HR in N. tabacum, N. clevelandii, or N. benthamiana (Table 1).

Agroinfiltration of the CymRSV $p 22 / p 19$ nested genes yielded an HR in N. edwardsonii and N. glutinosa, but initiation of the reaction was slower (6 to $7 \mathrm{dpi}$ ) than with either of the corresponding TBSV and CNV constructs. In addition, the HR induced by the CymRSV p22/p19 clone was variable, as sometimes only a portion of the infiltrated area became necrotic. With the TBSV and CNV nested clones, the entire infiltrated area consistently became necrotic within 4 dpi (Table 1). Agroinfiltration tests of individual CymRSV genes showed that the CymRSV p22 gene was responsible for triggering HR in N. edwardsonii and $N$. glutinosa but not involved in elicitation of HR in N. tabacum, N. clevelandii, or N. benthamiana.
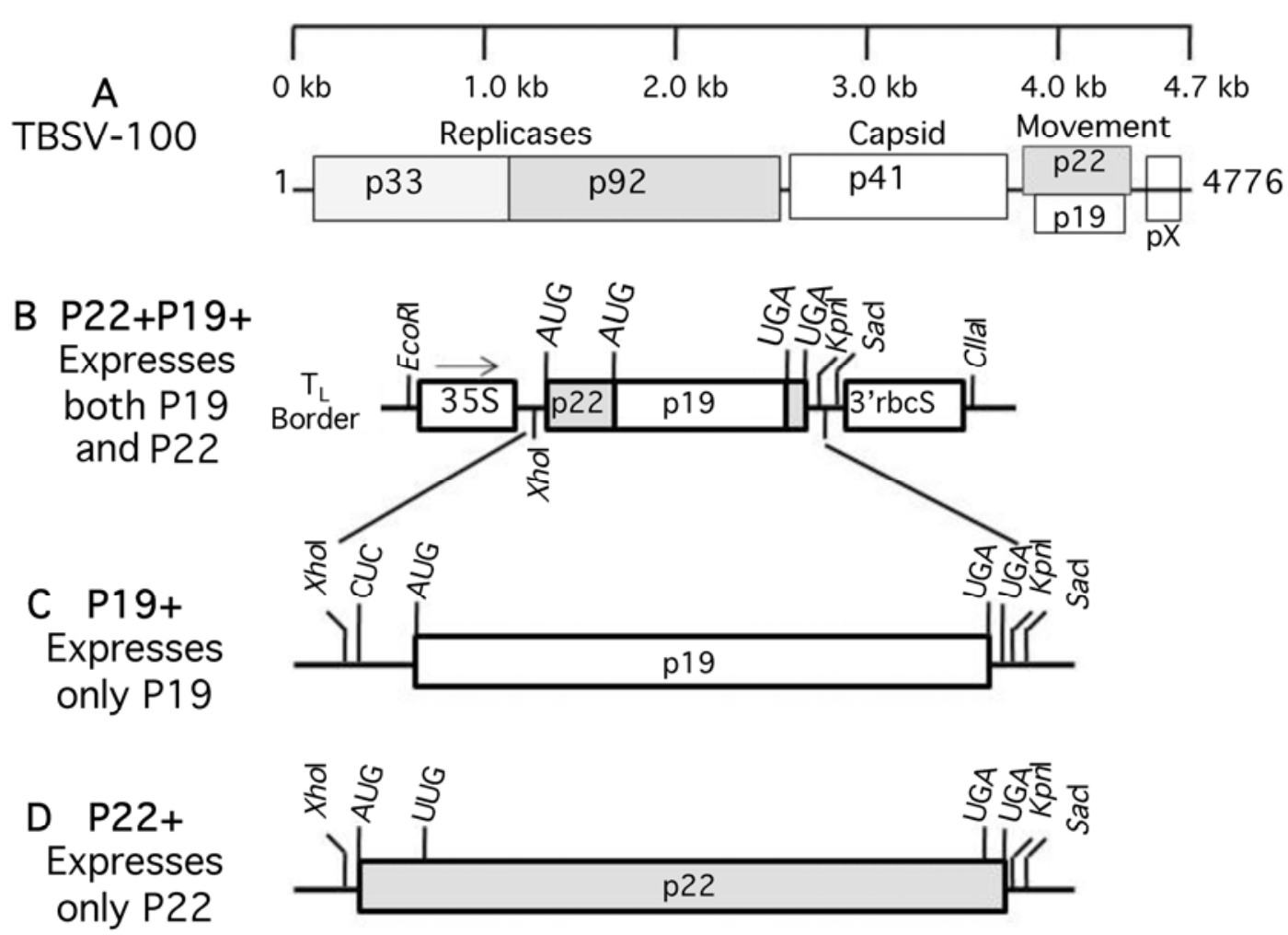
E P22-P19- Expresses neither P22 nor P19

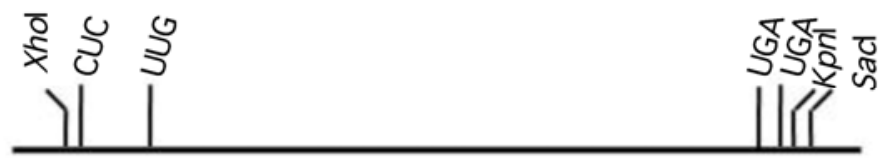

Fig. 1. Organization of the tombusvirus-Tomato bushy stunt virus (TBSV) genome and T-DNA constructs used for transient expression of tombusvirus genes in Agrobacterium tumefaciens vector pKYLX7. A, Diagram of TBSV genome. B, Insertion of tombusvirus sequences into pKYLX7. TBSV and CNV nested genes were cloned between the Xho I and Sac I sites, whereas CymRSV nested genes were cloned between XhoI and KpnI sites. C, Construct expresses only p19 (or in the case of CNV p20). D, Construct expresses only p22 (or in the case of CNV p21). E, Start codons for both $p 22$ and $p 19$ have been eliminated. 


\section{$N$. tabacum responds to agroinfiltration of the CymRSV p19 gene with HR, but agroinfiltration of CNV p20 fails to elicit HR.}

HR elicitation in N. tabacum was shown to be caused by the TBSV P19 protein. To determine if the analogous genes of CNV and CymRSV elicit HR in N. tabacum, we tested each of the $\mathrm{CNV}$ and CymRSV constructs described previously. Interestingly, neither the CNV nested $p 21 / p 20$ construct nor the CymRSV p22/p19 construct induced HR in N. tabacum within a period of 10 dpi (Supplementary Fig. 1). However, N. tabacum responded differently to infiltration of the CymRSV construct that expressed only the P19 protein, as this gene did elicit HR in $N$. tabacum within 3 dpi (Table 1). We considered that the inability of the CymRSV nested $p 21 / p 19$ construct to elicit HR in $N$. tabacum might be due to a lower level of translation of the $p 19$ gene in the nested construct relative to the construct that only expressed CymRSV $p 19$. In fact, mutations that alter the translational start site context of $p 22$ dramatically affected P19 protein levels (Scholthof et al. 1999), an indication that translation of the two proteins is tightly regulated in the nested construct.

By contrast, the construct that expressed only CNV P20 did not elicit HR (Table 1). We considered whether this construct itself might be defective, but CNV P20 did trigger an HR in another species, N. sylvestris. Furthermore, the silencing-suppres- sor function of CNV P20 was readily evident in an assay conducted in $N$. benthamiana. The silencing-suppressor strength of CNV P20 was indistinguishable from that of TBSV P19 and CymRSV P19 at 8 dpi, although it was weaker than the P19 pro-

Table 1. Response of Nicotiana species to agroinfiltration of tombusvirus P22 and P19 proteins ${ }^{\mathrm{a}}$

\begin{tabular}{lccc}
\hline Protein expressed & $\begin{array}{c}\boldsymbol{N} \text {. } \text { glutinosa } \text { and } \\
\text { N. edwardsonii }\end{array}$ & $\begin{array}{c}\text { N. benthamiana } \\
\text { and } \boldsymbol{N} \text {. clevelandii }\end{array}$ & $\begin{array}{c}\boldsymbol{N} . \\
\text { tabacum }\end{array}$ \\
\hline TBSV P22/P19 nested & HR & - & $\mathrm{HR}$ \\
TBSV P22 & HR & - & - \\
TBSV P19 & - & - & $\mathrm{HR}$ \\
TBSVP22/P19 KO & - & - & - \\
CNV P21/P20 nested & HR & - & - \\
CNV P21 & HR & - & - \\
CNV P20 & - & - & - \\
CNV P21/P20 KO & - & - & - \\
CymRSV P22/P19 & & - & - \\
nested & HR & - & - \\
CymRSV P22 & HR & - & HR \\
CymRSV P19 & - & - & - \\
CymRSV P22/P19 KO & - & - & \\
\hline
\end{tabular}

${ }^{\mathrm{a}} \mathrm{TBSV}=$ Tomato bushy stunt virus; $\mathrm{CNV}=$ Cucumber necrosis virus CymRSV = Cymbidium ringspot virus; $\mathrm{KO}=$ knock out; $\mathrm{HR}=$ hypersensitive response; $-=$ response to agroinfiltration is no different than the empty vector pKYLX7.
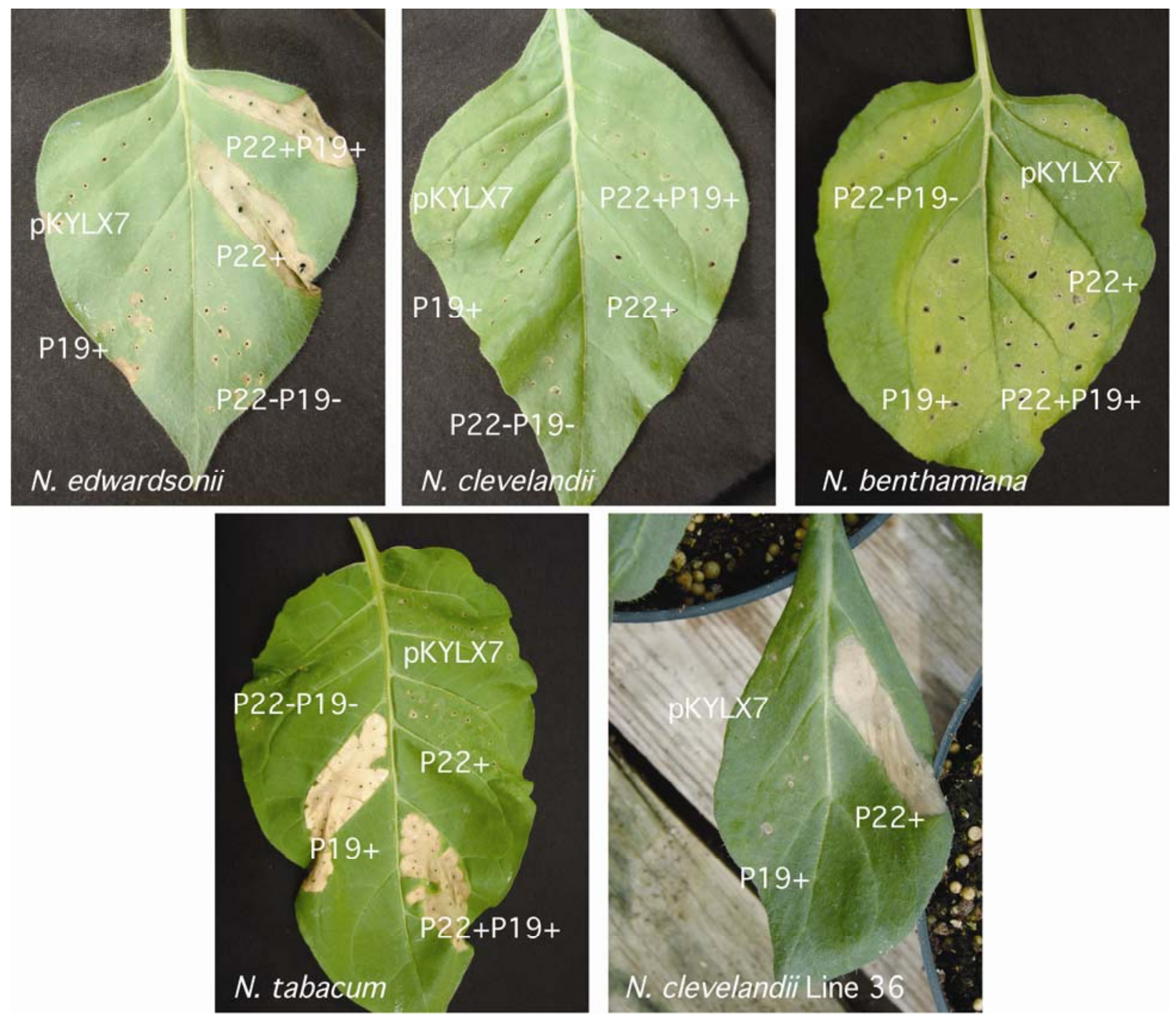

Fig. 2. Response of Nicotiana species to agroinfiltration of Tomato bushy stunt virus (TBSV) P19 and P22 proteins. All photographs were taken at 7 days postinfiltration. Leaf panels labeled pKYLX7 were agroinfiltrated with the empty vector. 
teins of TBSV and CymRSV at 12 dpi (Table 2). Taken together, these assays showed that the CNV p20 construct was functional and indicated that its inability to trigger HR in N. tabacum might be due to a unique feature of its amino acid sequence. A comparison of amino acid sequences of TBSV P19 (Supplementary Fig. 2), CymRSV P19, and CNV P20 showed that all three protein sequences were identical at $68 \%$ of the amino acid positions. However, CNV P20 and CymRSV P19 were more closely related to each other than to TBSV P19, and TBSV P19 is only $1 \%$ more closely related to CymRSV than to CNV P20. Conse- quently, there are 17 amino acid positions within the CNV P20 sequence that differ from TBSV P19 and CymRSV P19 that might explain its failure to elicit an HR in N. tabacum.

The observation that the CNV P20 protein did not trigger HR in N. tabacum suggested that the CNV virus might also not trigger an HR in this host. To investigate this possibility, CNV virions were inoculated to $N$. tabacum in a test that included TBSV and CymRSV virions. All three viruses elicited necrotic lesions by 5 dpi (Supplementary Fig. 3), and N. tabacum plants were resistant to infection. Agroinfiltration of CNV p21
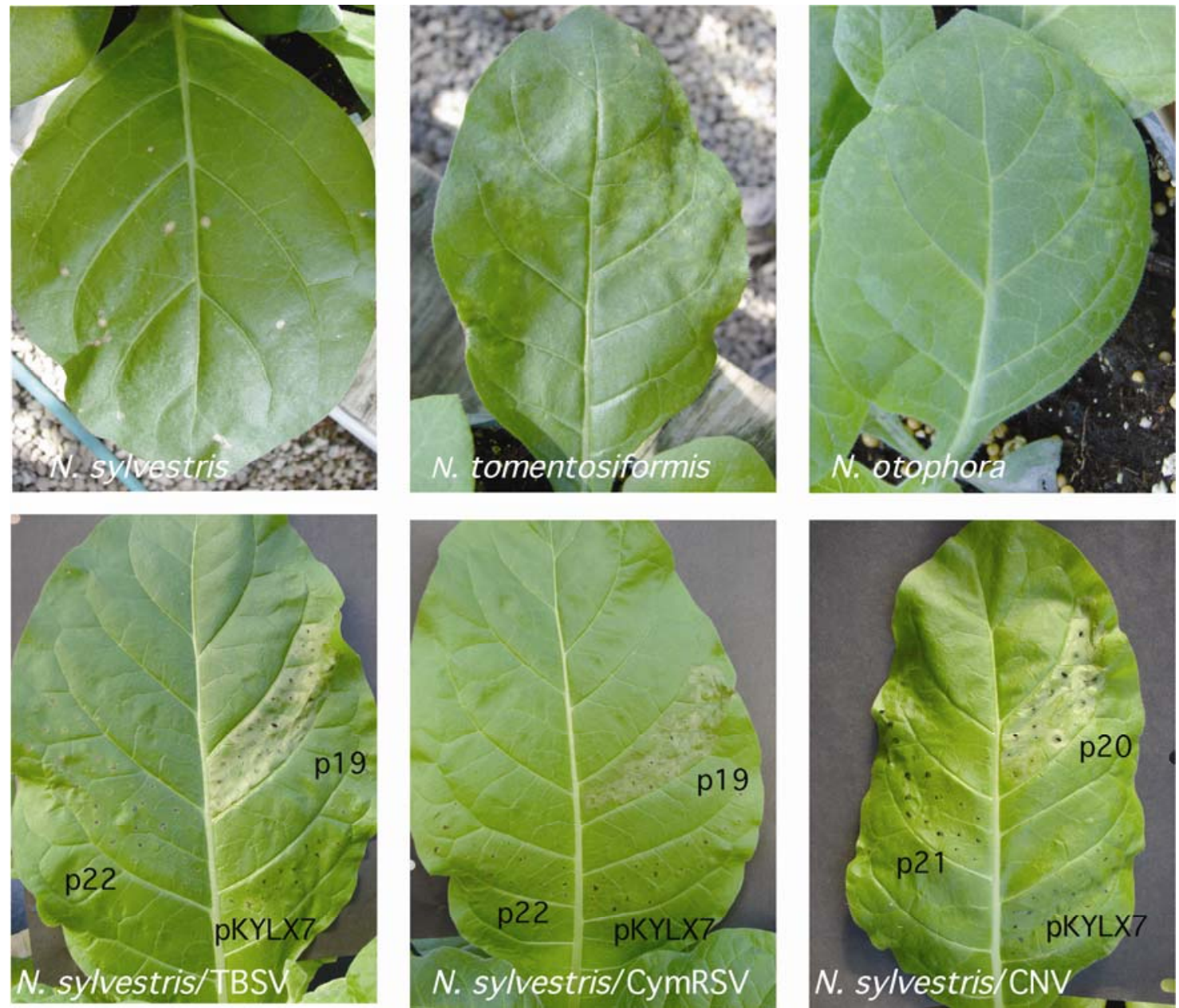

Fig. 3. Response of Nicotiana tabacum parents to tombusvirus virion inoculations and agroinfiltration of tombusvirus proteins. The top row illustrates the response of Nicotiana species to inoculation of Tomato bushy stunt virus (TBSV) at 5 days postinfiltration (dpi). The bottom row illustrates the response of N. sylvestris at 3 dpi to agroinfiltration of TBSV P19 and P22, CymRSV P19, and P22, and CNV P20 and P21.
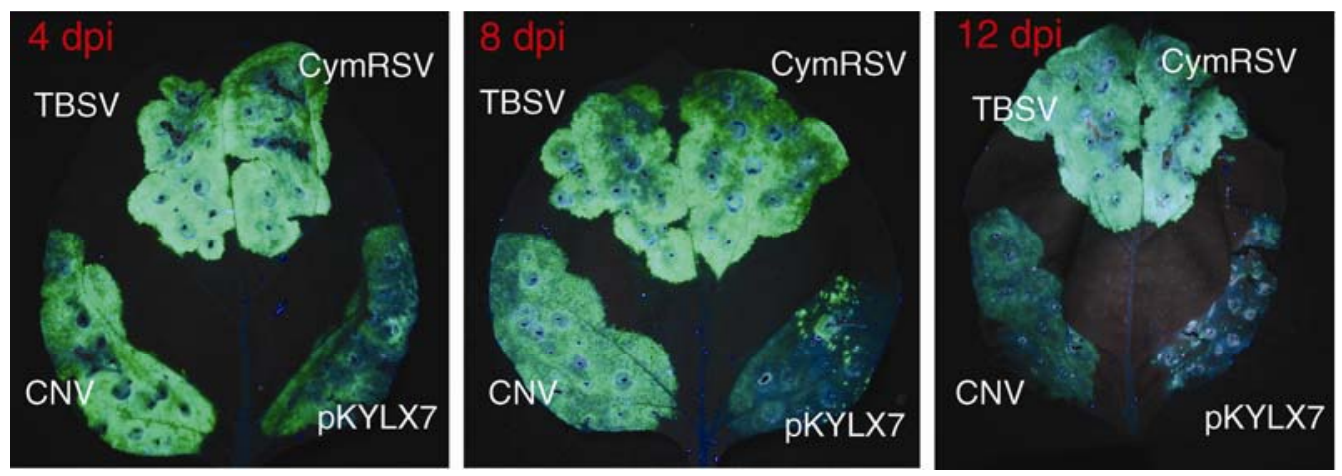

Fig. 4. Comparative strength of tombusvirus silencing suppressors. Green fluorescent protein (GFP) was coagroinfiltrated into Nicotiana benthamiana leaf panels with either Tomato bushy stunt virus (TBSV) p19, CymRSV p19, CNV p20, or the empty vector pKYLX7. GFP expression is illustrated at 4, 8, and 12 days postinfiltration. 
also did not induce HR, so at this time, it is still unclear which $\mathrm{CNV}$ protein is responsible for triggering the resistance response in N. tabacum.

\section{The HR elicited by TBSV P19}

in $N$. tabacum is inherited from its $N$. sylvestris parent.

The $N$. tabacum amphidiploid genome is derived primarily from N. sylvestris and N. tomentosiformis (Aoki and Ito 2002; Kovarik et al. 2004; Murad et al. 2002), but some studies have suggested that a portion of its genome is also acquired from $N$. otophora (Goodspeed 1954; Ren and Timko 2001; Riechers and Timko 1999). Since the parents of N. tabacum have been identified, this presented a unique opportunity to identify which parent might have contributed the putative $R$ gene in $N$. tabacum that recognizes the tombusvirus 19 gene to trigger an HR.

To identify which species might have contributed the putative $R$ gene, we initially inoculated $N$. tabacum and each of its three parents with virions of TBSV, CNV, and CymRSV. Only $N$. sylvestris (Fig. 3, TBSV illustrated) and $N$. tabacum responded to virion inoculations of the three viruses with HR. By contrast, $N$. tomentosiformis and $N$. otophora responded to the three viruses with chlorotic lesions that were most apparent at $5 \mathrm{dpi}$ (Fig. 3). Interestingly, none of the four Nicotiana species developed systemic symptoms in response to the three viruses. Furthermore, double-antibody sandwich-enzyme-linked immunosorbent assay (DAS-ELISA) tests conducted between 21 and 25 dpi showed that no virus could be detected in upper, noninoculated leaves (data not shown). Consequently, only the response of $N$. sylvestris to the tombusviruses was consistent with the HR observed in N. tabacum, although N. tomentosiformis and $N$. otophora did contain a nonnecrotic form of resistance.

Agroinfiltration of TBSV and CymRSV $p 19$ genes and the CNV p20 gene into the leaves of $N$. sylvestris, $N$. tomentosiformis, and $N$. otophora confirmed that $N$. sylvestris contained the putative $R$ gene. $N$. sylvestris responded to the agroinfiltration of the tombusvirus proteins with HR by 3 dpi (Fig. 3), whereas no reaction was observed in $N$. tomentosiformis and $N$. otophora (data not shown). Furthermore, no HR was observed in any of these Nicotiana species in response to the TBSV and CymRSV P22 proteins and CNV P21 protein (Fig. 3 ), which is consistent with the observation that $N$. tabacum does not contain a $R$ gene product that can recognize these tombusvirus P22 and P21 proteins. Agroinfiltration of CNV p20 into N. sylvestris yielded an HR (Fig. 3), by contrast to results obtained with CNV p2O and $N$. tabacum. These results suggest that, although the plant $R$ gene product that recognizes tombusvirus P19 proteins is derived from $N$. sylvestris, alleles may exist in $N$. tabacum species that can discriminate between CNV P20 and the other two tombusvirus proteins TBSV P19 and CymRSV P19.

Table 2. Green fluorescent protein (GFP) expression presented as a percentage of total protein ${ }^{\mathrm{a}}$

\begin{tabular}{lccc}
\hline & \multicolumn{3}{c}{ Percentage of GFP (mean \pm SD) } \\
\cline { 2 - 4 } Silencing suppressor & $\mathbf{4}$ dpi & $\mathbf{8 ~ d p i}$ & $\mathbf{1 2}$ dpi \\
\hline TBSV P19 & $4.88 \pm 1.08$ & $5.90 \pm 1.90$ & $4.12 \pm 1.26$ \\
CymRSV P19 & $6.08 \pm 2.73$ & $4.39 \pm 1.34$ & $4.08 \pm 2.14$ \\
CNV P20 & $5.87 \pm 0.34$ & $4.12 \pm 2.49$ & $1.68 \pm 0.27$ \\
pKYLX7 empty vector (control) & $4.44 \pm 1.08$ & $0.41 \pm 0.37$ & $0.31 \pm 0.53$ \\
\hline
\end{tabular}

${ }^{a}$ Obtained after coagroinfiltration into Nicotiana benthamiana leaves of a construct expressing GFP with one of three tombusvirus-silencing suppressors or the empty vector pKYLX7. TBSV = Tomato bushy stunt virus $;$ CymRSV $=$ Cymbidium ringspot virus $; \mathrm{CNV}=$ Cucumber necrosis virus; $\mathrm{SD}=$ standard deviation; $\mathrm{dpi}=$ days postinfiltration.

${ }^{\mathrm{b}}$ GFP was coagroinfiltrated in a 1:1 ratio with the silencing suppressor or with pKYLX7.

\section{Evaluation of the gene-silencing-suppression activity} of tombusvirus P19/P20 proteins.

Tombusvirus P19/P20 proteins have a specific role in suppression of gene silencing; specifically, TBSV P19 and CymRSV P19 proteins (Szittya et al. 2002; Voinnet et al. 1999). For instance, TBSV P19 has been shown to enhance the expression of GFP when coinfiltrated with GFP into $N$. benthamiana leaves (Voinnet et al. 1999, 2003). To investigate whether tombusvirus P19 proteins might differ in the strength of their silencing suppressors, we coagroinfiltrated TBSV $p 19$, CymRSV $p 19$, and CNV p20 with GFP and then assessed their ability to enhance and extend GFP expression. The treated leaves were observed for GFP fluorescence and protein accumulation. Representative photos of whole leaves are shown at 4, 8, and 12 dpi (Fig. 4), and GFP protein expressed as a percentage of total soluble protein is presented in Table 2.

As shown in Figure 4 and Table 2, maximal GFP expression obtained without coagroinfiltration of a tombusvirus P19 protein was seen at $4 \mathrm{dpi}$ and had diminished considerably by 8 dpi. By contrast, coinfiltration of the constructs expressing TBSV and CymRSV P19 and CNV P20 together with GFP suppressed RNA silencing; the degree of silencing suppression was equivalent for all three proteins at 8 dpi (Fig. 4; Table 2). When whole leaves were examined with a UV lamp at $12 \mathrm{dpi}$, CNV P20 was unable to sustain GFP expression to the same extent as TBSV P19 and CymRSV P19, which preserved the capacity to suppress gene silencing and extend expression of GFP for more than 20 days (data not shown). The GFP ELISA assay confirmed that the CNV P20 silencing suppressor remained functional at 12 dpi but was not as effective as either the TBSV or CymRSV P19 proteins.

\section{DISCUSSION}

Several studies have contributed to understanding the interactions of tombusvirus Avr proteins with putative R proteins in Nicotiana species. By using a PVX-based vector, Scholthof and associates (1995a) demonstrated that TBSV P22 protein elicited HR-like necrotic lesions in the TBSV-resistant hosts $N$. glutinosa and N. edwardsonii. However, N. glutinosa and $N$. edwardsonii did not remain resistant to the PVX-p22 construct, because the inherent instability of the PVX vector allowed for the deletion of the $p 22$ gene and, once it was eliminated, the PVX virus could spread unimpeded. Similarly, it was found that the TBSV P19 protein elicited HR-like lesions in N. tabacum and systemic necrosis in $N$. clevelandii and $N$. benthamiana (Scholthof et al. 1995a). It was also reported that, in addition to the P19 protein, the P33 protein of CymRSV contributed to the lethal necrosis phenotype in $N$. benthamiana infections (Burgyán et al. 2000). On the host side, resistance to TBSV was successfully introgressed from $N$. glutinosa to $N$. clevelandii, and it was shown that the $N$. clevelandii line developed in this cross (line 36) was also resistant to CymRSV and CNV (Schoelz et al. 2006). In addition, we showed that silencing of the resistance to TBSV in N. edwardsonii also had the effect of silencing resistance to CymRSV (Balaji et al. 2007). In the present study, we have investigated whether the P22 and P19 proteins of CymRSV and CNV would elicit the same response in resistant Nicotiana species as their TBSV counterparts.

We initially showed that agroinfiltration and expression of the TBSV P22 protein resulted in an HR at 3 to 4 dpi in both $N$. glutinosa and $N$. edwardsonii leaves and did not trigger any visible response in $N$. tabacum, $N$. benthamiana, or $N$. clevelandii. This result extends the observations of Scholthof and associates (1995a), because it shows HR does not occur through a synergistic interaction with other viral proteins but is solely dependent on expression of the P22 protein. The 
CymRSV P22 and CNV P21 proteins elicited the same responses as TBSV P22 in these Nicotiana species, although we did observe slight delays in HR development in response to the CymRSV P22 protein. An inspection of the TBSV P22 amino acid sequence revealed that it is $83 \%$ identical to CymRSV P22 and 86\% to CNV P21 (Supplementary Fig. 4). Furthermore, TBSV P22 amino acids shown to be critical for elicitation of HR by Chu and associates (1999), in particular amino acids 14 to 18 and 59, were conserved in the amino acid sequences of CymRSV P22 and CNV P21. These findings suggest that an $\mathrm{R}$ protein derived from $N$. glutinosa is able to recognize conserved features present in tombusvirus P22 proteins.

We found that agroinfiltration and expression of the TBSV P19 protein triggered HR in N. tabacum, which also was in agreement with Scholthof and associates (1995a). In our agroinfiltration tests of the CymRSV P19 and CNV P20 proteins, only CymRSV P19 triggered HR in N. tabacum, whereas CNV P20 did not elicit any response in that host. We also showed that CNV P20 was expressed; because it could elicit $\mathrm{HR}$ in $N$. sylvestris and also functioned as a silencing suppressor in $N$. benthamiana, its inability to trigger HR in $N$. tabacum must be related to differences between its amino acid sequence and those of TBSV and CymRSV. An alignment of CNV P20 with the P19 proteins of TBSV and CymRSV revealed 17 amino acid changes unique to the CNV P20 sequence that might result in its failure to elicit an HR in $N$. tabacum. In previous studies, point mutations introduced into the TBSV P19 protein abolished its capacity to elicit HR in N. tabacum (Chu et al. 2000; Hsieh et al. 2009). These modifications specifically targeted regions on P19 that were exposed on the surface of the protein and could be available for probable host-specific interactions as well as siRNA binding without altering the TBSV P22 protein sequence present in a different reading frame. In particular, Chu and associates (2000) found that individual mutations at amino acids 43, 71, 72 , the double mutant 75/78, and 85, each abolish HR in $N$. tabacum but have no effect on TBSV accumulation in $N$. benthamiana. However, none of the amino acids at these locations are unique to the CNV P20 protein, so none of them could be responsible for the abolishment of P19-induced necrosis in N. tabacum. Based on this, we considered that these amino acids are not responsible for the inability of CNV P20 to trigger HR in N. tabacum. Instead, it is likely that multiple mutations in $\mathrm{P} 19 / \mathrm{P} 20$ proteins may affect recognition by a putative $\mathrm{R}$ protein in N. tabacum.

We also found that agroinfiltration and expression of the tombusvirus P19 proteins in N. clevelandii and N. benthamiana leaves did not elicit a necrosis response in a timely manner. The P19 proteins did elicit weak necrosis in N. benthamiana leaves after $10 \mathrm{dpi}$, but this appeared to be conditioned by environmental conditions, especially low light intensity. The late responses of these Nicotiana species underscores that these plants are susceptible to tombusvirus infection, in contrast to the relatively rapid HR (within 3 to $5 \mathrm{dpi}$ ) that develops in resistant plants. Our results differed from Scholthof and associates (1995a), who found that P19 triggered a systemic necrosis symptom in $N$. clevelandii and $N$. benthamiana plants when the protein was expressed from a PVX vector. However our results are in agreement with Burgyán and associates (2000), who found that the systemic necrosis symptom in $N$. benthamiana was dependent on both the P33 protein and P19 proteins. It may be that the systemic necrosis observed by Scholthof and associates (1995a) was due to a synergism between TBSV P19 and one of the PVX proteins.

Our study also illustrates how agroinfiltration can be used to further characterize and track the origin of $R$ genes. Plant virolo- gists have accumulated a wealth of data on local lesion hosts, plant species that, in many cases, respond to virus infection with HR. In addition, the genomes of numerous plant viruses have also been characterized; in many instances, the number of proteins encoded in a viral genome is less than 10 and, for the majority of viruses, less than 20. Agroinfiltration provides a simple tool to rapidly survey a viral genome for the presence of Avr genes recognized by a host species that responds to the whole virus with HR.

In this regard, we used the available literature to examine the question of which parent of $N$. tabacum likely contributed the $R$ gene that triggered HR in response to the tombusvirus P19 protein? N. tabacum is derived mainly from N. sylvestris and $N$. tomentosiformis (Aoki and Ito 2002; Kovarik et al. 2004; Murad et al. 2002), although N. otophora is also thought to be a minor contributor (Goodspeed 1954; Ren and Timko 2001; Riechers and Timko 1999). We used a combination of virion inoculations and agroinfiltration assays to obtain evidence that a putative $R$ gene that recognizes the tombusvirus P19 protein in $N$. tabacum is derived from $N$. sylvestris. Agroinifltration as a tool for characterization of $R$ genes is not perfect. For example, we found that the CNV P20 protein did not elicit an HR in N. tabacum but did elicit an HR in N. sylvestris. It could be that the $R$ gene in the ancestral source of $N$. sylvestris that was incorporated into $N$. tabacum might have subtle differences in structure from the $R$ gene in the $N$. sylvestris that we used in our agroinfiltration studies. Alternatively, the genetic background of $N$. tabacum could exert subtle effects on the $R$ gene's capacity to recognize tombusvirus P19 proteins. This question likely will not be resolved until both $R$ genes are cloned and their structure compared, but we may gain some information in identifying the differences in the tombusvirus P19 protein sequences that led to the differential response in these Nicotiana species.

To compare the silencing-suppressor function of the tombusvirus P19 proteins, we coagroinfiltrated into $N$. benthamiana a construct expressing GFP with individual constructs expressing the TBSV and CymRSV p19 and the CNV p20 genes, as described by Voinnet and associates (2003). Interestingly, the silencing-suppression activities of the tombusvirus P19 proteins varied in strength and timing, as assessed by extending the expression of GFP in agroinfiltrated $N$. benthamiana leaf panels. Under our assay conditions, expression of GFP alone in N. benthamiana lasted for 5 to $7 \mathrm{dpi}$. At $8 \mathrm{dpi}$, the silencing-suppressor function of CNV P20 was equivalent to the P19 proteins of TBSV and CymRSV. We found that CymRSV P19 and TBSV P19 could extend GFP expression up to 20 days, whereas CNV P20 was weaker, as it could sustain GFP expression beyond 12 dpi, up to approximately $14 \mathrm{dpi}$. The revelation that P19 proteins differ in silencing-suppressor strength may ultimately be valuable for probing their role in silencing suppression.

\section{MATERIALS AND METHODS}

\section{Viruses and plants.}

Seeds of different Nicotiana species were obtained from the U.S. Tobacco Germplasm Collection at North Carolina State University (Lewis and Nicholson 2007) as follows: Nicotiana clevelandii (PI 555491), N. glutinosa (PI 555507), N. tabacum cvs. Turkish Samsun and NC95 (PI 552380), N. benthamiana (PI 555478), N. edwardsonii (PI 555704), N. sylvestris (PI $555569), N$. tomentosiformis (PI 555572), and N. otophora (PI 555542). To break dormancy, seeds were treated for $30 \mathrm{~min}$ with commercial bleach at $50 \%$ strength $(2.6 \% \mathrm{vol} / \mathrm{vol} \mathrm{NaOCl})$ (Burke 1957).

Tombusviruses TBSV, CNV, and CymRSV have all been previously cloned in infectious form (Grieco et al. 1989; Hearne 
et al. 1990; Rochon and Johnston 1991). Infectious, uncapped transcripts derived from the clones were made according to Scholthof and associates (1993) and were inoculated to either $N$. clevelandii or $N$. benthamiana to increase inoculum, and infected tissue was frozen for further inoculations. For inoculation of test plants, leaves infected with tombusviruses were ground with a mortar and pestle at a dilution of approximately 1:20 (wt/vol) with inoculation buffer $(0.05 \mathrm{M}$ phosphate buffer, $\mathrm{pH}$ 7.0) and then were gently rubbed onto Nicotiana leaves lightly dusted with 600-mesh Carborundum.

\section{ELISA tests for virus detection.}

To evaluate the capacity of tombusviruses to infect Nicotiana species, DAS-ELISA (Clark and Adams 1977) was performed using primary antibodies and alkaline phosphatase conjugates purchased from Agdia (Elkhart, IN, U.S.A.) for TBSV and CymRSV and from Acdia Inc. (Fayetteville, AR, U.S.A.) for CNV. Plant tissues were collected from upper noninoculated leaves between 20 and $28 \mathrm{dpi}$, and samples were ground at a ratio of 1:10 tissue/grinding buffer (1x phosphate buffered saline, $0.5 \%$ Tween $20,2 \%$ polyvinylpyrrolidone, molecular weight 40,000 , and $0.2 \%$ bovine serum albumin). Colorimetric reactions with the substrate p-nitrophenyl phosphate were quantified at $405 \mathrm{~nm}$ using a Multiskan MCC/340 microplate reader (Thermofisher Scientific, Cincinnati, OH, U.S.A.).

\section{Amplification and cloning of tombusvirus genes and the GFP coding sequence into the A. tumefaciens binary vector PKYLX7.}

PCR primers were developed from the nucleotide sequences of TBSV-cherry isolate (Hearne et al. 1990, National Center for Biotechnology Information [NCBI] accession number M21958), CNV (Rochon and Tremaine 1989; NCBI accession number M25270), and CymRSV (Grieco et al. 1989; NCBI accession number X15511). Primer sequences for PCR amplification were synthesized by Integrated DNA Technologies (Coralville, IA, U.S.A.). The forward primer for the TBSV p22/p19 and CNV p20/p21 nested genes was 5'-CTCGAGAT GGATACTGAATACGAACAAGTCAAT-3' and the reverse primer was 5'-GAGCTCTCAGACTGAAGAGTCTGTCTTAC TCG-3'. The forward primer for CymRSV p22/p19 nested genes was $5^{\prime}$-CTCGAGATGGACACTGAATACCAACAAGT T-3', and the reverse primer was 5'-GGTACCCTAGACTGAA GAGTCTGTCCTACT- 3 '. To knock out the $p 22$ genes of TBSV and CymRSV and the $p 21$ gene of CNV, the start codon AUG was mutated to CUC. The forward primer for TBSV and CNV was 5'-CTCGAGTTCCTCGATACTGAATACGAACAA-3' and the forward primer for CymRSV was 5'-CTCGAGTTCCTCG ACACTGAATACCAACAAGTT-3'.

To knock out the TBSV $p 19$, CymRSV p19, and CNV p20 genes, the start codon AUG was mutated to UUG, which conserved the amino acid sequence of the TBSV and CymRSV P22 proteins and the CNV P21 protein. The forward primer for TBSV and CNV clones was 5'-CTCGAGATGGATACTGAAT ACGAACAAGTCAATAAACCTTGGAAC-3' and for the CymRSV $p 19$ knockout was 5'-CTCGAGATGGACACTGAA TACCAACAAGTTAATAAACCTTGGAAC-3'. To construct the double knockouts, both start codons were changed using forward primers that incorporated the mutations of the single knockouts. Reverse primers for all knockouts were the same used for amplification of tombusvirus nested genes. PCR conditions were one cycle at $95^{\circ} \mathrm{C}$ for $2 \mathrm{~min}$, followed by $35 \mathrm{cy}$ cles at $95^{\circ} \mathrm{C}$ for $1 \mathrm{~min}, 62^{\circ} \mathrm{C}$ for $1 \mathrm{~min}$, and $72^{\circ} \mathrm{C}$ for $1 \mathrm{~min}$, and finally, one cycle at $72^{\circ} \mathrm{C}$ for $10 \mathrm{~min}$. The GFP coding sequence (Siemering et al. 1996) was amplified with the forward primer 5'-GGCCTCGAGATGAGTAAAGGAGAAGAACTTT
TCACT- $3^{\prime}$ and the reverse primer 5'-GGCCGGATCCGAGCT CTTATTTGTATAGTTCATCCATGCCATG-3'.

The amplified DNA was subsequently purified by agarose gel elution using a QIAquick gel extraction kit (Qiagen Inc., Valencia, CA, U.S.A.) and was cloned into the XhoI-SacI or XhoI$K p n I$ restriction enzyme sites of pGEM-7Zf(+) or into pGEM-T Easy (Promega Corp., Madison, WI, U.S.A.). Escherichia coli transformants were selected on Luria Bertani (LB) media containing kanamycin $(50 \mu \mathrm{g} / \mathrm{ml})$ and tetracycline $(12.5 \mu \mathrm{g} / \mathrm{ml})$ and were tested by colony PCR and restriction enzyme digestions for the presence of the tombusvirus insert. Nucleotide sequences of candidate clones were determined to confirm that the PCR reactions did not introduce errors. Sequencing of clones in both orientations was performed by the DNA Core Facility at the University of Missouri (Columbia, MO, U.S.A.).

After confirming the fidelity of all sequences, the tombusvirus and GFP inserts were subsequently cloned into the multiple cloning site of the Agrobacterium tumefaciens binary vector pKYLX7 (Schardl et al. 1987). Clones of pKYLX7 containing the tombusvirus and GFP genes were mobilized by electroporation (PG200 Progenetor II, Hoefer Scientific Instruments, San Francisco, CA, U.S.A.) into A. tumefaciens AGL1 (Lazo et al. 1991). Transformants were selected on LB medium supplemented with kanamycin $(50 \mu \mathrm{g} / \mathrm{ml})$, tetracycline $(12.5 \mu \mathrm{g} / \mathrm{ml})$, and carbenicillin $(50 \mu \mathrm{g} / \mathrm{ml})$.

\section{Agroinfiltrations.}

The induction and infiltration medium for experiments involving HR elicitation was $20 \mathrm{mM}$ MES [monohydrate 2-( $\mathrm{N}$ morpholino) ethanesulfonic acid], $60 \mathrm{mM}$ sucrose, $55 \mathrm{mM}$ glucose, $\mathrm{pH} 5.4$, and $2 \mathrm{mM}$ acetosyringone dissolved into $\mathrm{N}-\mathrm{N}$ dimethylformamide. For experiments involving coagroinfiltrations of GFP and tombusvirus $p 19$ genes, the medium for overnight induction contained $200 \mu \mathrm{M}$ MES, $\mathrm{pH} 5.85$, and 19.5 $\mu \mathrm{M}$ acetosyringone. The final optical density at $600 \mathrm{~nm}$ of agrobacteria containing each plasmid was 1.0. The infiltration buffer included $10 \mathrm{mM}$ MES (pH 5.85), $10 \mathrm{mM} \mathrm{MgCl}_{2}$, and $2.25 \mathrm{mM}$ acetosyringone. Agroinfiltrations were done on the abaxial surface of fully expanded leaves of 4- to 8-week Nicotiana spp., using a needleless plastic syringe. Coagroinfiltrations for silencing-suppression experiments were done in $N$. benthamiana only. Every experiment consisted of four agroinfiltrated leaves per plant and two plants per replica. The entire experiment was repeated at least three times.

\section{GFP ELISA.}

Plant samples were initially ground in a $50 \mathrm{mM}$ phosphate buffer $(\mathrm{pH}$ 7.0), and total protein was determined by a Bradford assay (Bio Rad, Hercules, CA, U.S.A.). The GFP ELISA was conducted using a kit from Cell Biolabs (San Diego, CA, U.S.A.). Briefly, $250 \mathrm{pg}$ of total protein was loaded into each well of a microtiter plate precoated with GFP antibodies and was incubated according to the manufacturer's directions. In subsequent steps, the wells were incubated with an anti-GFP antibody, a second antibody conjugated to horseradish peroxidase, and the substrate provided in the kit. The absorbance obtained from the reaction was converted to picograms of GFP per milliliter, using a standard curve that ranged from 15.6 to $1,000 \mathrm{pg} \mathrm{GFP} / \mathrm{ml}$. The GFP protein values obtained for each timepoint were the average of three separate samples, and the GFP ELISA was repeated more than three times.

\section{ACKNOWLEDGMENTS}

The authors thank H. Scholthof for gifts of TBSV antisera and for helpful comments on the manuscript. This research was supported by the Missouri Agricultural Experiment Station. 


\section{LITERATURE CITED}

Aoki, S., and Ito, M. 2000. Molecular phylogeny of Nicotiana (Solanaceae) based on the nucleotide sequence of the matK gene. Plant Biol. 2:316-324.

Balaji, B., Cawly, J., Angel, C., Zhang, Z., Palanichelvam, K., Cole, A., and Schoelz, J. 2007. Silencing of the $N$ family of resistance genes in Nicotiana edwardsonii compromises the hypersensitive response to tombusviruses. Mol. Plant-Microbe Interact. 20:1262-1270.

Bhattacharjee, S., Zamora, A., Azhar, M. T., Sacco, M. A., Lambert, L. H., and Moffett, P. 2009. Virus resistance induced by NB-LRR proteins involves argonaute4-dependent translational control. Plant J. 58:94-951.

Burgyán, J., Hornyik, C., Szittya, G., Silhavy, D., and Bisztray, G. 2000. The ORF1 products of tombusviruses play a crucial role in lethal necrosis of virus-infected plants. J. Virol. 74:10873-10881.

Burke, L. G. 1957. Overcoming seed dormancy in Nicotiana. Agron. J. 49:461.

Christie, S. R. 1969. Nicotiana hybrid developed as a host for plant viruses. Plant Dis. Rep. 53:939-941.

Chu, M., Park, J.-W., and Scholthof, H. B. 1999. Separate regions on the Tomato bushy stunt virus P22 protein mediate cell-to-cell movement versus elicitation of effective resistance responses. Mol. Plant-Microbe Interact. 12:285-292.

Chu, M., Desvoyes, B., Turina, M., Noad, R., and Scholthof, H. B. 2000. Genetic dissection of Tomato bushy stunt virus P19-protein-mediated host-dependent symptom induction and systemic invasion. Virology, 266:79-87.

Clark, M. F., and Adams, N. A. 1977. Characteristics of the microplate method of enzyme-linked immunosorbent assay for the detection of plant viruses. J. Gen. Virol. 34:475-483.

Culver, J. N., and Dawson, W. O. 1991. Tobacco mosaic virus elicitor coat protein genes produce a hypersensitive phenotype in transgenic Nicotiana sylvestris plants. Mol. Plant-Microbe Interact. 4:458-463.

Daubert, S. D., Schoelz, J. E., Debao, L., and Shepherd, R. J. 1984. Expression of disease symptoms in cauliflower mosaic virus genomic hybrids. J. Mol. Appl. Genet. 2:537-547.

Desvoyes, B., and Scholthof, H. B. 2002. Host-dependent recombination of a Tomato bushy stunt virus coat protein mutant yields truncated capsid subunits that form virus-like complexes which benefit systemic spread. Virology 304:434-442.

Erickson, F., Holzberg, S., Calderon-Urrea, A., Handley, V., Axtell, M., Corr, C., and Baker, B. 1999. The helicase domain of the TMV replicase proteins induces the $\mathrm{N}$-mediated defense response in tobacco. Plant J. 18:67-75.

Flor, H. H. 1971. Current status of gene-for-gene concept. Annu. Rev. Phytopathol. 9:275-296.

Gerik, J. S., Duffues, J. E., Perry, R., Stenger, D. C., and Van Maren, A. F. 1990. Etiology of Tomato plant decline in the California desert. Phytopathology 80:1352-1356.

Goodspeed, T. H. 1954. The genus Nicotiana. Chronica Botanica, Walthman, Mass., USA. 536 p.

Grieco, F., Burgyán, J., and Russo, M. 1989. The nucleotide sequence of Cymbidium ringspot virus RNA. Nucleic Acids Res. 17:6383-6383.

Hearne, P. Q., Knorr, D. A., Hillman, B. I., and Morris, T. J. 1990. The complete genome structure and synthesis of infectious RNA from clones of Tomato bushy stunt virus. Virology 177:141-151.

Hsieh, Y.-C., Omarov, R. T., and Scholthof, H. B. 2009. Diverse and newly recognized effects associated with short interfering RNA binding site modifications on the Tomato bushy stunt virus P19 silencing suppressor. J. Virol. 83:2188-2200.

Kang, B.-C., Yeam, I., and Jahn, M. M. 2005. Genetics of plant virus resistance. Annu. Rev. Phytopathol. 43:581-622

Kovarik, A., Matyasek, R., Lim, K. Y., Skalická, K., Koukalová, B., Knapp, S., Chase, M., and Leitch, A. R. 2004. Concerted evolution of 18-5.8-26S rDNA repeats in Nicotiana allotetraploids. Biol. J. Linn. Soc. 82:615-625.

Lazo, G. R., Stein, P. A., and Ludwig, R. A. 1991 A DNA transformationcompetent Arabidopsis genomic library in Agrobacterium. BioTechnology 9:963-967.

Lewis, R. S., and Nicholson, J. S. 2007. Aspects of the evolution of Nicotiana tabacum L. and the status of the United States Nicotiana Germplasm Collection. Genet. Resour. Crop Ev. 54:727-740.

Murad, L., Lim, K. Y., Christopodulou, V., Matyasek, R., Lichtenstein, C. P., Kovarik, A., and Leitch, A. R. 2002. The origin of tobacco's T genome is traced to a particular lineage within Nicotiana tomentosiformis (Solanaceae) Am. J. Bot. 89:921-928

Nagy, P. D., and J. Pogany. 2008. Multiple roles of viral replication proteins in plant RNA virus replication. Methods Mol. Biol. 451:55-68.
Obermeier, C., Sears, J. L., Liu, H. Y., Schlueter, K. O., Ryder, E. J. Duffus, J. E., Koike, S. T., and Wisler, G. C. 2001. Characterization of distinct tombusviruses that cause diseases of lettuce and tomato in the western United States. Phytopathology 91:797-806.

Qu, F., and Morris, T. J. 2002. Efficient infection of Nicotiana benthamiana by Tomato bushy stunt virus is facilitated by the coat protein and maintained by $\mathrm{p} 19$ through suppression of gene silencing. Mol. PlantMicrobe Interact. 15:193-202.

Ren, N., and Timko, M. P. 2001. AFLP analysis of genetic polymorphism and evolutionary relationships among cultivated and wild Nicotiana species. Genome 44:559-571.

Riechers, D. E., and Timko, M. P. 1999. Structure and expression of the gene family encoding putrescine $\mathrm{N}$-methyltransferase in Nicotiana tabacum: New clues to the evolutionary origin of cultivated tobacco. Plant Mol. Biol. 41:387-401.

Rochon, D. M., and Johnston, J. C. 1991. Infectious transcripts from cloned Cucumber necrosis virus cDNA: Evidence for a bifunctional subgenomic mRNA. Virology 181:656-665.

Rochon, D. M., and Tremaine, J. H. 1989. Complete nucleotide sequence of the cucumber necrosis virus genome. Virology 169:251-259.

Schardl, C. L., Byrd, A. D., Benzion, G., Altschuler, M. A., Hildebrand, D. F., and Hunt, A. G. 1987. Design and construction of a versatile system for the expression of foreign genes in plants. Gene 61:1-11.

Schoelz, J. E., Shepherd, R. J., and Daubert, S. 1986. Region VI of Cauliflower mosaic virus encodes a host range determinant. Mol. Cell. Biol. 6:2632-2637

Schoelz, J. E., Wiggins, B. E., Wintermantel, W. M., and Ross, K. 2006. Introgression of a tombusvirus resistance locus from $N$. edwardsonii var. Columbia to $N$. clevelandii. Phytopathology 96:453-459.

Schoelz, J. E. 2006. Viral determinants of resistance versus susceptibility. Page 13-42 in: Natural Resistance Mechanisms of Plants to Viruses, G Loebenstein and J. P.Carr, eds. Springer, Dordrecht, The Netherlands.

Scholthof, H. B. 2006. The tombusvirus-encoded P19: From irrelevance to elegance. Nat. Rev. Microbiol. 4:405-411.

Scholthof, H. B., and Jackson, A. O. 1997. The enigma of pX: A host dependent cis-acting element with variable effects on tombusvirus RNA accumulation. Virology 237:56-65.

Scholthof, H. B., Scholthof K.-B. G., and Jackson A. O. 1995a. Identification of Tomato bushy stunt virus host-specific symptom determinants by expression of individual genes from a Potato virus $X$ vector. Plant Cell $7: 1157-1172$

Scholthof, H. B., Scholthof K.-B. G., and Jackson A. O. 1995b. The tomato bushy stunt virus replicase proteins are coordinately expressed and membrane associated. Virology 208:365-369.

Scholthof, H. B., Scholthof, K.-B. G., Kikkert, M., and Jackson, A. O. 1995c. Tomato bushy stunt virus spread is regulated by two nested genes that function in cell-to-cell movement and host-dependent systemic invasion. Virology 213:425-438.

Scholthof, H. B., Desvoyes, B., Kueker, J., and Whitehead, E. 1999. Biological activity of two tombusvirus proteins translated from nested genes is influenced by dosage control via context-dependent leaky scanning. Mol. Plant-Microbe. Interact. 12:670-679.

Scholtof, H. B., Morris, T. J., and Jackson, A. O. 1993. The capsid protein gene of Tomato bushy stunt virus is dispensable for systemic movement and can be replaced for localized expression of foreign genes. Mol. Plant-Microbe Interact. 6:309-322.

Siemering, K. R., Golbik, R., Sever, R., and Haseloff, J. 1996. Mutations that suppress the thermosensitivity of green fluorescent protein. Curr. Biol. 6:1653-1663.

Szittya, G., Molnár, A., Silhavy, D., Hornyik, C., and Burgyán, J. 2002. Short defective interfering RNAs of Tombusviruses are not targeted but trigger post-transcriptional gene silencing against their helper virus. Plant Cell, 14:359-372.

Voinnet, O., Pinto, Y. M., and Baulcombe, D. C. 1999. Suppression of gene silencing: A general strategy used by diverse DNA and RNA viruses. Proc. Natl. Acad. Sci. U.S.A. 96:14147-14152.

Voinnet, O., Rivas, S., Mestre, P., and Baulcombe, D. C. 2003. An enhanced transient expression system in plants based on suppression of gene silencing by the P19 protein of Tomato bushy stunt virus. Plant J. 33:949-956.

White, K. A., and Nagy, P. D. 2004. Advances in the molecular biology of tombusviruses: Gene expression, genome replication and recombination. Prog. Nucleic Acid Re. 78:187-226.

Whitham, S., Dinesh-Kumar, S. P., Choi, D., Hehl, R., Corr, C., and Baker, B. 1994. The product of the tobacco mosaic virus resistance gene $\mathrm{N}$ : Similarity to toll and the interleukin-1 receptor. Cell 78:1101-1115.

Yamamura, Y., and Scholthof, H.B. 2005. Pathogen profile-Tomato bushy stunt virus: A resilient model system for studying virus-plant interactions. Mol. Plant Pathol. 6:491-502. 